\title{
Study protocol: Evaluation of the Allergic Rhinitis Clinical Management Pathway (AR-CMaP) in Australian community pharmacies
}

\author{
Rachel Tan ${ }^{1}$, Vicky Kritikos ${ }^{1}$, Biljana Cvetkovski ${ }^{1}$, Janet Rimmer ${ }^{2}$, Kwok Yan ${ }^{3}$, Lynn \\ Cheong $^{4}$, Jean Bousquet ${ }^{5}$, and Sinthia Bosnic-Anticevich ${ }^{1}$ \\ ${ }^{1}$ Woolcock Institute of Medical Research \\ ${ }^{2}$ The University of Sydney Sydney Medical School \\ ${ }^{3}$ Royal Prince Alfred Hospital \\ ${ }^{4}$ University of Canberra \\ ${ }^{5}$ Centre Hospitalier Universitaire de Montpellier
}

May 15, 2020

\begin{abstract}
Allergic Rhinitis (AR) is a high burden chronic respiratory disease(1-4) affecting 19\% of Australians; $29 \%$ in the Australian Capital Territory(ACT), Australia. Up to $70 \%$ of people with AR self-select their medication in Australian pharmacies; with only $15 \%$ selecting optimal medication(6). The Allergic Rhinitis Clinical Management Pathway(AR-CMaP) was developed as an evidence-based AR management guide to support pharmacists to optimise AR management in the pharmacy. This paper describes the method used to investigate the implementation of AR-CMaP by evaluating the impact of AR-CMaP on AR medication management and pharmacists' practice and identifying the challenges associated with implementing AR-CMaP. This study took a mixed methods approach. The AR-CMaP was implemented and evaluated in a cohort of pharmacies in the ACT. Prior to the implementation of AR-CMaP, baseline data were collected in the pharmacy; pharmacists completed a needs assessment and a researcher administered questionnaires to people who purchased an AR-related product. The completed needs assessments individualised the AR-CMaP training and support tools provided to each participating pharmacy. Following pharmacists training, the AR-CMaP was implemented, pharmacists completed a second needs assessment and the questionnaire to people with AR were re-administered. Pharmacists were interviewed after all data collection was completed. There is an urgent need to evaluate the AR management services in community pharmacies. This study is the first to implement and evaluate an evidence-based clinical pathway in the pharmacy setting, in real life. Important insights into the practical aspects of AR management and clinical frameworks in the community pharmacy will be identified.
\end{abstract}

\section{Hosted file}

AR-CMaP Protocol_Manuscript.pdf available at https://authorea.com/users/322400/articles/ 451385-study-protocol-evaluation-of-the-allergic-rhinitis-clinical-management-pathwayar-cmap-in-australian-community-pharmacies 